\title{
The effect of solar wind structures on the storm-time magnetosphere
}

\author{
Maha Ashour-Abdalla ${ }^{1}$, M. El-Alaoui ${ }^{1}$, V. Peroomian ${ }^{1}$, \\ and R. J. Walker ${ }^{1}$ \\ ${ }^{1}$ Institute of Geophysics and Planetary Physics, \\ University of California, Los Angeles, CA 90095-1567, USA
}

\begin{abstract}
We have examined the effect of small- and large-scale structures in the solar wind on the topology and dynamics of the Earth's magnetosphere during magnetic storms. To do so, we ran global magnetohydrodynamic (MHD) simulations of a magnetic storm that occurred on 2324 May 2000 by using upstream solar wind data from the ACE, IMP-8, and Wind spacecraft to run separate simulations of the global magnetosphere. This study suggests that the presence of small-scale structures in the solar wind can affect the global configuration of the magnetosphere.
\end{abstract}

\section{Introduction}

The long term goal of NASA's Living With a Star program and the National Space Weather campaign is to forecast the dynamical state of the magnetosphere-ionosphere (M-I) system from satellite measurements made in the upstream solar wind (usually at the L1 Lagrange point, $220 R_{E}$ upstream of Earth) that are used to drive linear/nonlinear algorithms which predict the various geomagnetic indices (e.g. McPherron 1991; Vassiliadis et al. 1999) and/or global MHD simulations which predict the M-I plasma state and electrodynamic fields. The quality of predictions produced by these models are strongly dependent on the accuracy of the solar wind data that is used as input.

This paper examines the validity of assuming that the solar wind observed at L1 is a good predictor of the solar wind environment near the Earth, and evaluates the possible sources of error caused by the location of the solar wind monitor. We do so by selecting a magnetic storm during which more than one solar wind monitor was available, and by using MHD simulations driven by input from these monitors to highlight the similarities, differences, correlations, and significance of the solar wind observations.

\section{Results}

\subsection{The 23-24 May 2000 Event}

Three spacecraft were located in the solar wind during the 23-24 May 2000 magnetic storm: ACE was at the L1 point $\sim 220 R_{E}$ upstream, Wind was $\sim 60 R_{E}$ upstream, while IMP-8 was $\sim 30 R_{E}$ upstream. We have divided our study into two intervals. The first, from 2200 on 23 May to 0600 on 24 May covers the interval when the magnetic cloud was passing the Earth. The second interval, from 0900 UT to 1500 UT on 24 May was in the wake of the cloud. Figure 1 shows the three components of the IMF and the total magnetic field for the two intervals. In this figure, we used the sharp northward turning of the IMF at 09:45 UT to line up the three spacecraft. In order to line up all three traces shown in Figure 1, we used the Wind spacecraft (black curve) as a baseline, then shifted the ACE measurements (red curves) forward in time by 31:32 min, and the IMP-8 results (green curves) backward in time by 9:34 min. This was done so that we could 

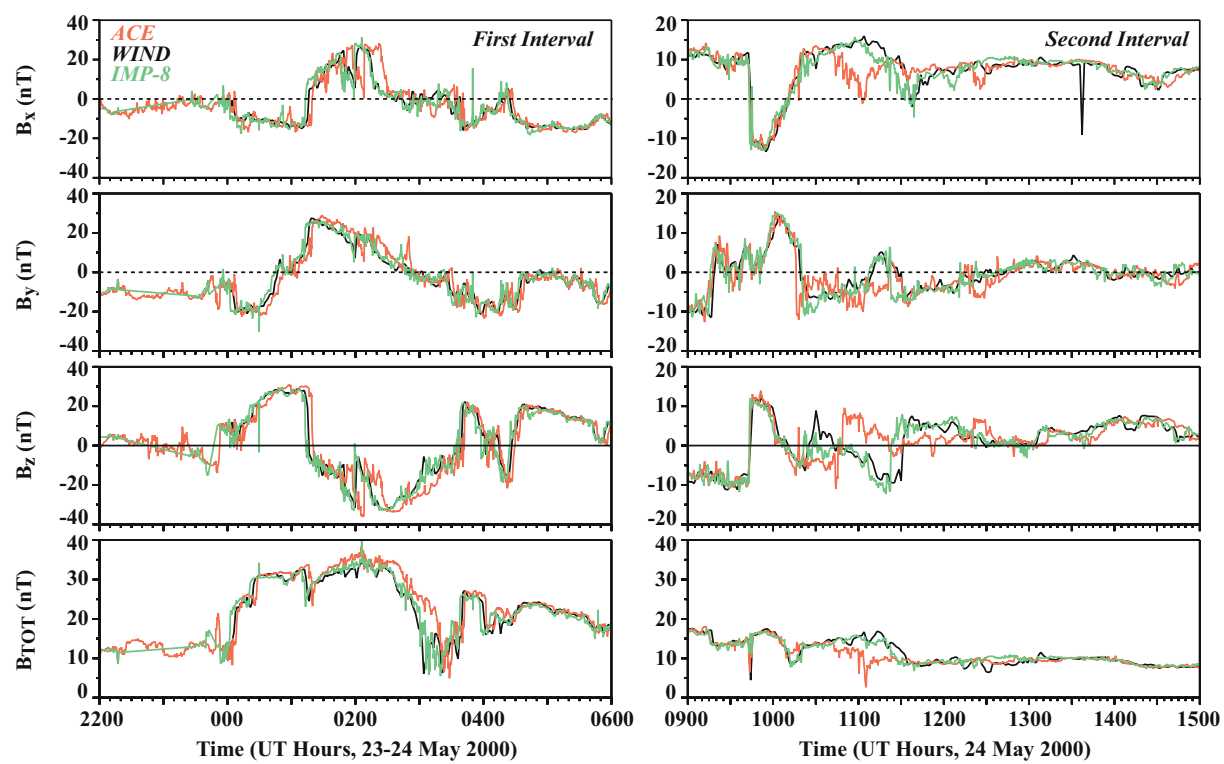

Figure 1. Three components of the IMF and total $|\boldsymbol{B}|$ for (left-hand column) the first time interval, and (right-hand column) the second interval.

examine the effect of the assumption of the ballistic propagation of the solar wind from the monitor to the Earth. Figure 1 shows that the IMF observed by all three spacecraft was qualitatively the same during the first interval (left-hand panels), but that during the second interval (right-hand panels), ACE observed a northward turning of the IMF and an overall reduction in the IMF $|\boldsymbol{B}|$ at $\sim 10: 45$ UT which was not observed by the two spacecraft nearer Earth.

\subsection{IMF Cross-Correlations}

We have carried out a cross-correlation analysis of the solar wind observations from ACE, Wind and IMP-8. In Figure 2 we have plotted the maximum cross-correlation of IMF $\mathrm{B}_{z}$ for the three pairs of observations Wind and IMP-8, Wind and ACE, and IMP-8 and ACE. The observed $\mathrm{B}_{z}$ in GSE coordinates is at the bottom of the figure. We filtered the observations by using a 10-min running average in order to eliminate the effects of fine-scale structure in the IMF time series. This was done so that we could determine the differences in the observed solar wind as a function of frequency. We found that frequencies faster than $10 \mathrm{~min}$ account for most of the variance in the first interval, but do not account for the large-scale differences in the second interval. One would not expect these high-frequency differences to significantly affect the magnetosphere since they are faster than the time it takes for flux to convect over the dayside magnetosphere. The MHD simulations shown in Figure 3 bear out these findings. Throughout the first interval there is excellent agreement between the three monitors. The worst is between Wind and ACE ( 90\%) and the best is between Wind and IMP-8 ( 95\%). However, starting at about $0600 \mathrm{UT}$, the cross-correlations get much worse, especially between $\mathrm{ACE}$ and the two spacecraft nearer the Earth. Examination of the actual data traces on the bottom shows large-scale agreement until about 1000 UT. After this time, the low peak cross-correlations represent large-scale structures in the time series. Note that the peak cross-correlation between Wind and IMP-8, the monitors closest to the Earth, is between $80 \%$ and $90 \%$ during this interval compared with $\sim 95 \%$ prior to 0600 UT. 


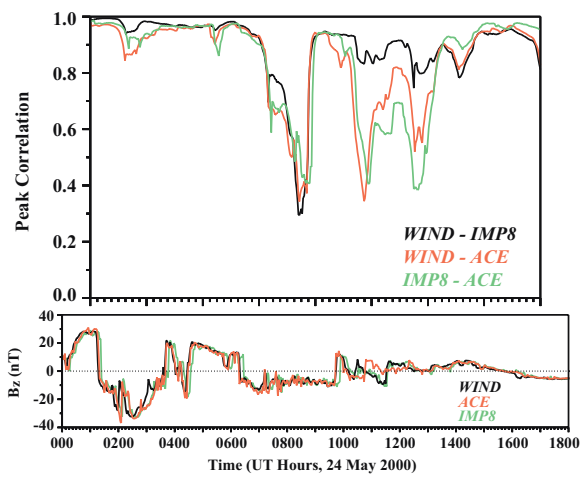

Figure 2. a) Cross-correlations of IMF $\mathrm{B}_{z}$ for 10-min averaged data.
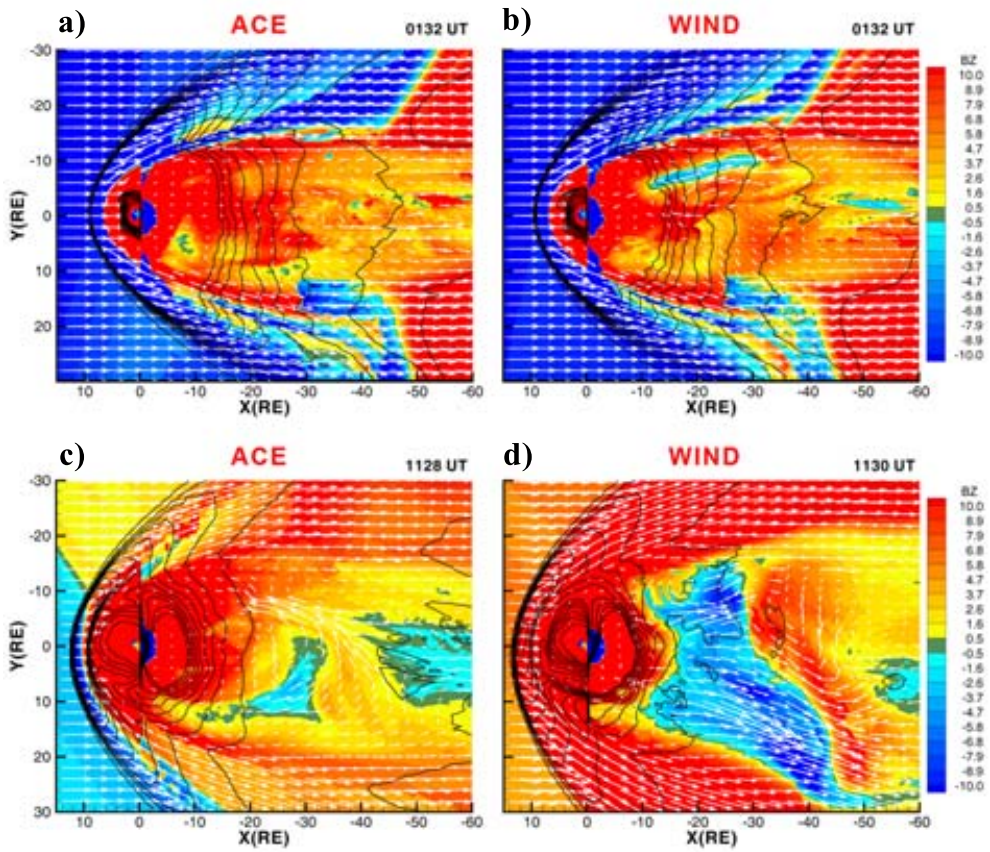

Figure 3. Snapshots of the equatorial plane from the MHD simulations driven by the (left-hand) ACE data, and (right-hand) Wind data at (a and b) 0132 UT during the first interval and (c and d) at 1132 UT during the second interval. The color contours show the Bz in the simulations, the black curves are isocontours of MHD pressure, and the white arrows show the flow direction.

\subsection{Simulation Results}

For this part of the study, we used measurements from ACE and Wind as input to our global MHD simulation model and carried out two separate simulations of the magnetosphere (see Raeder et al. 2001 and El-Alaoui 2001 for details of the MHD simulation model). Figure 3 shows results from these simulations at 0132 UT during the main phase (panels a and b) and at $1132 \mathrm{UT}$ during the early recovery phase (panels $\mathrm{c}$ and $\mathrm{d}$ ). Figure 3 shows that during the main phase of the storm, the magnetospheric configuration was similar in the two simulations, but not identical. On the other hand, during the early recovery phase, the overall configuration of the magnetosphere was significantly different for large periods of time (Figure $3 \mathrm{c}$ and $3 \mathrm{~d}$ ). 

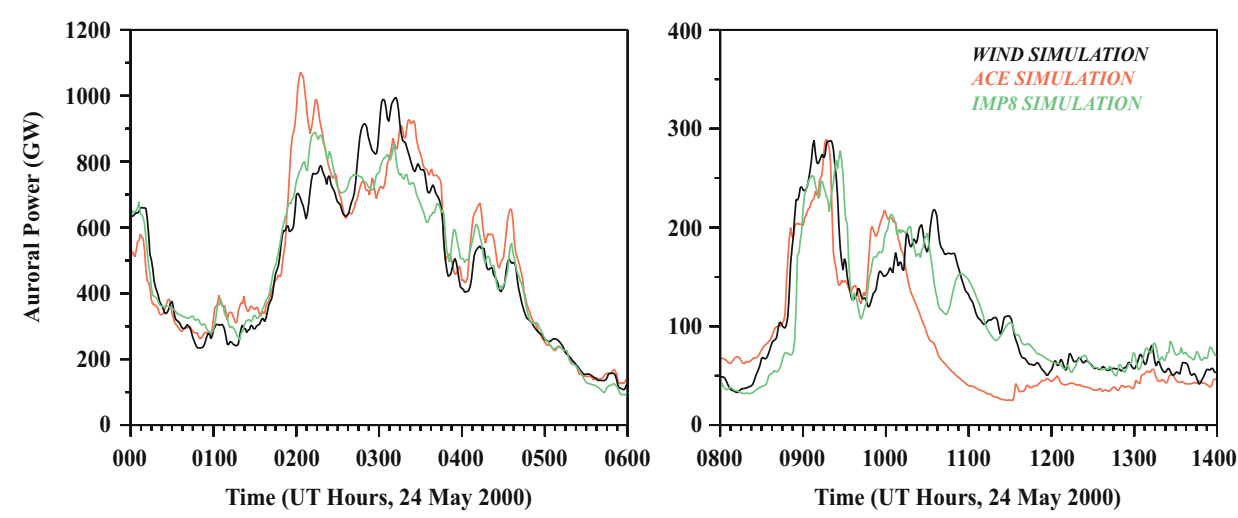

Figure 4. (Power deposited into the ionosphere for simulations driven by the ACE (red), Wind (black) and IMP-8 (green) data for the (a) first and (b) second intervals.

Figure 4 shows a comparison of the power deposited into the ionosphere for the MHD simulations drived by the ACE, Wind, and IMP-8 data for both intervals. The Wind and IMP-8 results are qualitatively similar, but not identical. Figure 4 clearly shows the effect of the northward turning observed only by the ACE spacecraft. The power deposited into the ionosphere and the cross-polar-cap potential are significantly lower for the ACE simulation after 10:45 UT.

\section{Conclusions}

Our simulations of the 23-24 May 2000 magnetic storm have shown that:

1. Early in the storm, observations from the three solar wind monitors were consistent and the simulated magnetospheres were similar. The cross-correlations were very sensitive to the higher frequency variations in the plasma parameters but the simulated magnetospheres were not. The magnetosphere is a low pass filter.

2. Later in the storm the observations at the three solar wind monitors differed greatly as did the simulated magnetospheres. The largest differences were between ACE and the other monitors but there were significant differences between Wind and IMP-8 nearer the Earth as well. The three monitors were not observing the same solar wind.

We therefore conclude that the magnetic cloud (first interval) is a coherent structure on spatial scales important for predicting the magnetospheric configuration. However, the second interval is incoherent and the location of solar wind measurement dramatically changes the configuration and dynamics of the simulated magnetosphere.

\section{Acknowledgements}

This study was supported by NSF grant ATM-0208507. Computing support was provided by the San Diego Supercomputer Center.

\section{References}

El-Alaoui, M. 2001, J. Geophys. Res., 106, 6229.

Raeder, J., McPherron, R.L., Frank, L. A., Kokubun, S., Lu, G., Mukai, T., Paterson, W. R., Sigwarth, J. B., Singer, H. J., \& Slavin, J. A. 2001, J. Geophys. Res., 106, 381.

McPherron, R. L. 1991, Geomagnetism, 4, 593.

Vassiliadis, D., Klimas, A. J., Valdivia, J. A., \& Baker, D. N. 1999, J. Geophys. Res., 104, $24,957$. 